\title{
ESTUDO ECOLÓGICO: PERFIL DA MORBIMORTALIDADE POR HANSENÍASE NO BRASIL ENTRE 2015 E 2019
}

Guilherme Ribeiro Ferreira, Anna Luiza Rodrigues Brito, Isabela dos Santos Rodolfo, Rafaela Spolador Orbolato

Universidade do Oeste Paulista - UNOESTE, Presidente Prudente, SP. E-mail: guisharkboy@gmail.com

\section{RESUMO}

A hanseníase é uma doença crônica, infecciosa, transmitida através da inalação de aerossóis contendo Mycobacterium leprae. O presente estudo foi feito para investigar o perfil epidemiológico da hanseníase no Brasil entre 2015 e 2019, avaliando as taxas de prevalência de internações hospitalares, incidência, saída por abandono, entrada por recidiva e mortalidades. Trata-se de um estudo ecológico conduzido a partir de dados coletados em sistemas de informações disponíveis no Departamento de Informática do Sistema Único de Saúde (DATASUS). No intervalo de tempo considerado foi evidenciada uma discreta melhora nos indicadores de saúde investigados. Diante disso, é possível inferir que o país fez um manejo adequado da hanseníase entre 2015 e 2019, provavelmente por realizar boas medidas profiláticas, boas intervenções e conscientização eficiente dos infectados.

Palavras-chave: hanseníase, indicadores de morbimortalidade, perfil de saúde, incidência, mortalidade.

\section{ECOLOGICAL STUDY: MORBIDITY AND MORTALITY PROFILE DUE TO LEPROSY IN BRAZIL BETWEEN 2015 AND 2019}

\begin{abstract}
Leprosy is a chronic, infectious disease transmitted through the inhalation of aerosols containing Mycobacterium leprae. The present study was carried out to investigate the epidemiological profile of leprosy in Brazil between 2015 and 2019, evaluating the prevalence rate of hospital admissions, incidence rate, number of admissions due to recurrence and mortality rates. It is an ecological study conducted from data collected in information systems available at the Departamento de Informática do Sistema Único de Saúde (DATASUS). In the considered time interval, a slight improvement in the investigated health indicators was evidenced. It is possible to infer that the country carried out an adequate management of leprosy between 2015 and 2019, probably because of good prophylactic actions, good interventions and efficient awareness of those infected.
\end{abstract}

Keywords: leprosy, indicators of morbidity and mortality, health profile, incidence, mortality.

\section{INTRODUÇÃO}

A hanseníase é uma doença crônica, infecciosa, transmitida através da inalação de aerossóis contendo Mycobacterium leprae. Esse microrganismo é um bacilo álcool-ácido resistente que se multiplica lentamente, sobrevive por dias fora do hospedeiro, tem um período de incubação prolongado e, graças a isso, sua infecção pode levar até 20 anos para produzir sintomas e desenvolver uma doença de fato ${ }^{1}$.
M. leprae infecta principalmente as células de Schwann presentes em nervos periféricos, mas pode afetar também mucosas e outros órgãos como os olhos, ossos e fígado. Os principais sinais e sintomas da hanseníase são manchas esbranquiçadas ou avermelhadas na pele com alterações de sensibilidade térmica, dolorosa e tátil; pápulas, tubérculos e nódulos pelo corpo; câimbras, dormências e perda de sensibilidade periférica, principalmente em mãos 
e pés; queda dos pêlos, diminuição da força muscular, edema de mãos e pés associado a cianose e ressecamento da pele ${ }^{2}$.

As principais complicações de um manejo incorreto da hanseníase são neuropatias e alterações de pele permanentes, deficiências físicas, paralisias musculares e deformidades ósseas. É comum que os pacientes sem acompanhamento adequado tenham lesões repetidas em mãos e pés graças à anestesia que a infecção provoca, isso leva a danos irreversíveis que podem gerar a necessidade de amputação do membro. As incapacidades físicas na hanseníase podem sinalizar o diagnóstico tardio, indiretamente refletindo o nível de conscientização da população sobre os sinais e sintomas da doença, o acesso aos serviços de saúde e a habilidade das equipes em fazer o diagnóstico e tratamento precoces ${ }^{3}$.

O diagnóstico do paciente hanseniano se baseia na anamnese e exame físico, porém pode ser confirmado com exames complementares como baciloscopia de raspado intradérmico e biópsia de pele. $O$ exame físico é iniciado pela pesquisa de lesões cutâneas por todo o corpo, avaliação dos nervos cutâneos e sensibilidade dos diversos territórios da pele ${ }^{2}$.

É essencial que, durante toda a consulta, o médico enfatize que hanseníase tem cura e que o tratamento é gratuito pelo Sistema Único de Saúde (SUS), auxilie o paciente a eliminar sentimentos de preconceito, alerte sobre as complicações e esclareça todas as dúvidas que forem levantadas. A instrução acerca dos efeitos colaterais das drogas usadas no esquema terapêutico é indispensável para evitar desistências e falhas no tratamento ${ }^{2}$.

No momento do diagnóstico de hanseníase, é feita a classificação operacional do caso de acordo com o número de lesões cutâneas. Hanseníase Paubacilar (PB) é aquela em que o paciente apresenta até cinco lesões de pele, acima disso caracteriza-se um quadro de Hanseníase Multibacilar (MB). A Hanseníase PB é chamada de "indeterminada" quando o paciente apresenta até cinco lesões com contornos mal definidos e sem comprometimento neural, ou "tuberculóide" quando existem até cinco lesões com contornos bem definidos, lesão neural e fibrose. Além dessa classificação, é possível caracterizar a infecção de acordo com a forma de apresentação das lesões. A Hanseníase na forma virchowiana é caracterizada por infiltrado de macrófagos e muitos bacilos em seu interior e no citoplasma das células de Schwann. Quando se tem um quadro de hanseníase tuberculóide na forma virchowiana, a nomenclatura dada é "hanseníase dimorfa". Vale mencionar que uma forma de diagnóstico da hanseníase quando as lesões cutâneas não estão bem definidas e de avaliar a efetividade do tratamento implementado é a biópsia de nervo, principalmente sural, radial superficial ou ramo dorsal do ulnar ${ }^{3}$.

Após identificar um paciente com hanseníase é necessário realizar o rastreio de contatos. Toda e qualquer pessoa que resida ou teve contato próximo e prolongado com o doente nos últimos cinco anos anteriores ao diagnóstico deve ser submetida à anamnese dirigida, exame dermatoneurológico e uma dose de vacina BCG (exceto em pessoas com tuberculose ativa, imunossuprimidos ou gestantes) cuja finalidade não é imunizar, mas proporcionar maior resistência ao organismo do contato, principalmente contra a Hanseníase MB. Recomenda-se que os contatos sejam submetidos a anamnese dirigida e exame dermatoneurológico anualmente, por um período de cinco anos ${ }^{2,4}$.

O tratamento gratuito para hanseníase oferecido pelo SUS é baseado na poliquimioterapia (PQT) feita com Rifampicina, Dapsona e Clofazimina. Na primeira consulta, logo após o diagnóstico, já se inicia o tratamento, caso não existam contraindicações, para curar o paciente e interromper a cadeia de transmissão o quanto antes. Após completar o tratamento o paciente é considerado curado, mesmo assim deve continuar realizando acompanhamento, especialmente quando a doença produziu complicações irreversíveis².

Pacientes adultos com hanseníase PB receberão um tratamento de seis meses, composto por uma dose mensal supervisionada de 600 mg de Rifampicina e 100 mg diárias de Dapsona que devem ser tomadas em casa. Por outro lado, adultos com hanseníase MB devem ser tratados por doze meses com uma dose mensal de $600 \mathrm{mg}$ de Rifampicina, $100 \mathrm{mg}$ de Dapsona e 300 mg de Clofazimina. Em domicílio, esses pacientes devem tomar diariamente 100 $\mathrm{mg}$ de Dapsona e $50 \mathrm{mg}$ de Clofazimina ${ }^{2}$.

Desde os tempos bíblicos, a hanseníase é uma doença de grande importância para a saúde pública. Pela possibilidade de produzir paralisias, atrofias, incapacidades físicas e deformidades, por muito tempo hansenianos sofreram 
preconceito e exclusão social. Atualmente essa condição melhorou, porém o auto-julgamento e a auto-segregação que o infectado pratica ainda são problemas relevantes ${ }^{5}$.

Em 2019, de acordo com dados da World Health Organization, o Brasil foi considerado o segundo país com maior incidência de hanseníase em todo o mundo, contabilizando um total de 27.863 novos casos, ficando atrás apenas da Índia com 114.451 novos casos $^{6}$. Considerando a população brasileira neste ano, é possível afirmar que $1,3 \mathrm{em}$ cada $10 \mathrm{mil}$ pessoas foram diagnosticadas com hanseníase ${ }^{7}$. No mundo estão registrados 192.713 casos de hanseníase, correspondendo à taxa de prevalência global de 0,25/10.000 habitantes, e uma detecção de 210.617 casos novos ao ano (taxa de 2,77/100.000 habitantes), detecção considerada de média intensidade ${ }^{8}$.

Ao avaliar o intervalo de tempo entre 2015 e 2020 é possível notar uma taxa de abandono do tratamento para hanseníase considerável. Dos 155.902 diagnosticados nesse período, $6,8 \%$ abandonaram o tratamento ${ }^{9}$. Além de favorecer a resistência bacteriana e o surgimento de complicações, o abandono do tratamento faz com que a cadeia de transmissão permaneça ativa e que recidivas ocorram mais facilmente. Por conta dessa e das outras informações supracitadas, traçar o perfil epidemiológico da hanseníase no país é válido para que seja possível identificar a necessidade de implantar ou aprimorar políticas públicas de saúde voltadas à essa infecção.

O presente estudo foi conduzido para investigar o perfil da morbidade e mortalidade por hanseníase no Brasil entre 2015 e 2019, enfatizando índices de incidência, taxa de abandono, recidiva e mortalidade. A partir do delineamento e avaliação de tais indicadores de saúde, identificar se as políticas públicas de prevenção, diagnóstico e tratamento para hanseníase foram eficazes e aprimoradas no período avaliado.

\section{MÉTODOS}

Estudo ecológico conduzido a partir de dados coletados em sistemas de informações disponíveis no Departamento de Informática do Sistema Único de Saúde (DATASUS). As bases de dados usadas foram o Sistema de Informação de Agravos de Notificação (Sinan), Sistema de Informações Hospitalares do SUS (SIH/ SUS), Sistema de Informações sobre Mortalidade e Instituto Brasileiro de Geografia e Estatística (IBGE)/ Gerência de Estudos e Análises da Dinâmica Demográfica.

Foi considerado todo o território nacional, os anos de 2015 a 2019 como critérios de elegibilidade para os dados buscados, como critério de exclusão foi levado em conta informações ignoradas porque elas poderiam superestimar os resultados finais.

A avaliação do perfil epidemiológico da hanseníase foi baseada nos números de entradas por recidiva, saídas por abandono, taxas de prevalência e incidência, além das taxas de mortalidade específica e proporcional da doença. Os dados usados para calcular as taxas foram o número de óbitos por ano, novos casos diagnosticados em cada ano, total de casos a cada ano, população total a cada ano, entrada por recidiva e saída por abandono.

Todos os cálculos foram feitos por dois pesquisadores e comparados posteriormente para evitar possíveis erros. A partir disso, foi feita a análise estatística dos indicadores calculados a partir da comparação das taxas encontradas a cada ano.

\section{RESULTADOS}

Os números de entradas por recidiva, saídas por abandono e as taxas de prevalência e incidência (ambas feitas na base $10^{5}$ ) de hanseníase no Brasil estão representados na tabela a seguir. 
Tabela 1. Número de entradas por recidiva e saídas por abandono, taxa de prevalência e taxa de incidência de hanseníase no Brasil por ano, entre 2015 e 2019.

\begin{tabular}{|c|c|c|c|c|}
\hline Ano & $\begin{array}{c}\text { Entradas por } \\
\text { recidiva }\end{array}$ & $\begin{array}{c}\text { Saídas por } \\
\text { abandono }\end{array}$ & $\begin{array}{c}\text { Taxa de } \\
\text { prevalência }\end{array}$ & Taxa de incidência \\
\hline 2015 & 1574 & 2331 & 17,57 & 14,44 \\
\hline 2016 & 1454 & 2103 & 15,63 & 12,60 \\
\hline 2017 & 1663 & 2240 & 16,69 & 13,22 \\
\hline 2018 & 1856 & 2120 & 17,68 & 13,97 \\
\hline 2019 & 1713 & 1640 & 17,18 & 13,48 \\
\hline
\end{tabular}

Quanto às taxas de mortalidade, foi identificada taxa de mortalidade proporcional igual a $0,04 \%$ em todos os anos avaliados, já a taxa de mortalidade específica (base $10^{6}$ ) sofreu pequenas variações ao longo do tempo, sendo sempre menor do que 3 mortes a cada 1 milhão de brasileiros ${ }^{10}$.

\section{DISCUSSÃO}

As taxas de incidência de hanseníase no Brasil apresentaram melhora entre 2015 e 2019, sendo possível relacionar o menor número de novos casos com uma menor transmissão da doença, possivelmente proporcionada por melhorias na educação em saúde da população e nos serviços de atenção primária à saúde.

Os indicadores de mortalidade específica e proporcional não demonstraram alterações relevantes e permaneceram baixos, permitindo inferir que o manejo dos casos existentes foi eficiente e que o risco de uma pessoa morrer por hanseníase é baixo. Tal comportamento contrasta com a literatura existente, uma vez que a hanseníase é considerada um problema de saúde pública no país por sua mortalidade em determinadas regiões do país que têm alta endemicidade, como Norte e Nordeste ${ }^{11}$.

Dentre todas as informações coletadas, o destaque deve ser dado ao número de entradas por recidivas e saídas por abandono, uma vez que ambas reduziram entre o início e o fim do período estudado, fato que demonstra que a conscientização da população sobre a importância da adesão e seguimento ao tratamento foi eficiente, mesmo se tratando de uma doença de difícil enfrentamento e tratamento de difícil adesão.

A hanseníase é uma doença crônica, cuja infecção pode levar até 20 anos para produzir sintomas e desenvolver uma doença de fato. Considerando o impacto psicológico, biológico e epidemiológico dessa doença, fez-se necessário conhecer o perfil da morbimortalidade dela no Brasil, uma vez que, de acordo com a World Health Organization, foi o segundo país com maior incidência de hanseníase em 2019 em todo o mundo ${ }^{6}$.

Quanto ao perfil de morbidade por hanseníase, notam-se menor incidência e prevalência de internações hospitalares em 2019 quando comparadas com as de 2015. O perfil da mortalidade por esta infecção foi caracterizado por menores taxas de mortalidade específica e uma taxa constante de mortalidade proporcional considerando os dois extremos do intervalo de tempo avaliado. Destaca-se também a redução das taxas de recidiva e abandono no período estudado, podendo supor a partir disso que a conscientização, a adesão ao tratamento e os resultados que ele produziu foram satisfatórios.

Tendo em vista as pequenas diferenças nos indicadores investigados, é possível inferir que houve uma discreta melhora no perfil da morbimortalidade por hanseníase no Brasil entre os anos de 2015 e 2019, o que indica boas medidas profiláticas que permitiram a redução da incidência, boas intervenções e conscientização eficiente dos infectados para que a taxa de mortalidade específica e o número de saída por abandono diminuíssem.

Conclui-se, portanto, que o Brasil realizou um manejo adequado da hanseníase entre 2015 e 2019 e que esse manejo foi aprimorado ao longo do tempo, possibilitando a redução da maioria dos indicadores em saúde avaliados, desta forma, mesmo sendo o 2 o país do mundo com maior taxa de incidência, foi capaz de enfrentar a hanseníase de maneira cada vez mais eficiente entre os anos investigados.

\section{CONFLITO DE INTERESSE}

Os autores declaram não haver qualquer potencial conflito de interesse que possa 
interferir na imparcialidade deste trabalho científico.

\section{REFERÊNCIAS}

1. Murray PR, Rosenthal KS, Pfaller MA. Microbiologia médica. 7. ed. Rio de Janeiro: Elsevier; 2014.

2. Ministério da Saúde (Brasil). Secretaria de Vigilância em Saúde. Departamento de Vigilância das Doenças Transmissíveis. Guia prático sobre a hanseníase [Internet]. 1. ed. Brasília: Ministério da Saúde; 2017 [citado em 2022 jan. 05]. Disponível em: http://bvsms.saude.gov.br/bvs/publicacoes/guia pratico hanseniase.pdf

3. Brasileiro Filho G. Bogliolo: patologia. 9. ed. Rio de Janeiro: Guanabara Koogan; 2016.

4. Nunes JM, Oliveira EN, Vieira NFC. Hanseníase: conhecimentos e mudanças na vida das pessoas acometidas. Ciên. Saúde Colet. 2011;16:13111318. DOI: https://doi.org/10.1590/S1413$\underline{81232011000700065}$

5. Eidt LM. Breve história da hanseníase: sua expansão do mundo para as Américas, o Brasil e o Rio Grande do Sul e sua trajetória na saúde pública brasileira. Saude soc. 2004;13(2):76-88. DOI: $\quad$ https://doi.org/10.1590/S0104$\underline{12902004000200008}$

6. World Health Organization. Global leprosy (Hansen disease) update, 2019: time to step-up prevention initiatives. Weekly epidemiological record. 2020 [cited 2022 jan. 05];36:417-440. Available from: https://apps.who.int/iris/bitstream/handle/1066 5/334140/WER9536-eng-

fre.pdf?sequence $=1$ \&isAllowed=y\&ua $=1$

7. Instituto Brasileiro de Geografia e Estatística. Diretoria de Pesquisas. Coordenação de População e Indicadores Sociais. Projeção da população do Brasil para o período de 2000-2030 [Internet]. Rio de Janeiro; 2021 [citado em 2022 jan. 05]. Disponível em: http://tabnet.datasus.gov.br/cgi/deftohtm.exe?i $\underline{\text { bge/cnv/projpopuf.def }}$

8. Marzliak MLC. Hanseníase: O controle da doença e desafios atuais. Bol. epidemiol. paul. 2019 [citado em 2022 jan. 05]; 16(182):37-44.
Disponível

em:

https://docs.bvsalud.org/biblioref/2019/10/1023 245/1518237-44.pdf

9. Ministério da Saúde (Brasil). Sistema de Informação de Agravos de Notificação [Internet]. Brasília; 2020 [citado em 2022 jan. 05]. Disponível em:

http://tabnet.datasus.gov.br/cgi/tabcgi.exe?sina nnet/cnv/hanswbr.def

10. Ministério da Saúde (Brasil). Sistema de Informações sobre Mortalidade [Internet]. Bras2021 [citado em 2022 jan. 05]. Disponível em:

http://tabnet.datasus.gov.br/cgi/deftohtm.exe?si $\underline{\mathrm{m} / \mathrm{cnv} / \text { obt10uf.def }}$

11. Ferreira AF, Souza EA, Lima MS, García GSM, Corona F, Andrade ESN, et al. Mortalidade por hanseníase em contextos de alta endemicidade: análise espaço-temporal integrada no Brasil. Rev Panam Salud Publica. 2019;43:1-10. DOI: https://doi.org/10.26633/RPSP.2019.87 